\title{
Mitigation of Terrestrial Radar Interference in L-Band Spaceborne Microwave Radiometers
}

\author{
J.R. Piepmeier and F.A. Pellerano \\ Microwave Instrument Technology Branch \\ NASA's Goddard Space Flight Center \\ Greenbelt, MD 21146 \\ Email: jeff.piepmeier@nasa.gov
}

\begin{abstract}
Terrestrial radars operating in the 1215-1400 $\mathrm{MHz}$ radio-location and navigation spectrum allocation are important for air traffic safety, homeland security, and national defense. For low-frequency observations of soil moisture and ocean salinity, Earth-observing microwave radiometers are allocated EarthExploration Satellite Service (EESS) spectrum for operating at $1400-1427 \mathrm{MHz}$. The proximity of powerful long-range radars to the passive allocation makes observing a challenge. Three aspects of mitigation to RFI are discussed in this paper: survivability, operability, and excisability (SOE). Modeling and simulations of NASA's Hydros and Aquarius radiometers were performed to examine the impacts of radar interference. The results are applied to the three aspects of mitigation SOE and the affects on the radiometer requirements are discussed.
\end{abstract}

\section{INTRODUCTION}

The physics of microwave thermal emission dictate that low frequencies be used for radiometers to measure ocean-surface salinity and soil moisture (through any reasonable amount of vegetation). The Earth Exploration Satellite Service (EESS) enjoys an exclusive passive allocation at 1400-1427 MHz, a band in which transmission is prohibited [1]. Two L-band microwave radiometers will be launched into orbit before the decade's end. (It was three until NASA canceled it's Hydros mission [2] to measure global soil moisture and freeze-thaw state - see http://hydros.gsfc.nasa.gov). NASA's Aquarius radiometer will measure ocean surface salinity on a global scale [3] and ESA's SMOS mission will measure soil moisture [4]. If radiometer receivers where perfectly selective to their allocated band and neighboring transmitters had perfect control of outof-band (OOB) emissions, radio-frequency interference (RFI) would not be an issue. Experience shows, however, this is not the case [5]-[8]. Since no L-band radiometers have flown in space since Skylab, airborne and ground-based experience augmented by analysis must be used to predict the potential impact of the spectrum environment. By experience, the most problematic interference is due to terrestrial radars TR's and a previous analysis for SMOS also predicts this to be the case [9].

In this paper, the impacts of interference due to TR's operating below $1400 \mathrm{MHz}$ on the engineering requirements of Aquarius and Hydros are analyzed. The RFI analyses herein are from a study of TR's commissioned by the NASA Earth Science Spectrum Management Office for the Aquarius and
Hydros missions [10]. Given the impacts, three aspects to mitigation are proposed: survivability, operability, and excisability; or SOE. Survivability deals with avoiding damage from RFI. This means proper filtering and limiting. A radiometer achieves operability when it can measure, without error, the antenna or brightness temperature in the presence of interference. Selective receivers operating in quite spectrum are required for operability. When filtering and frequency selection is not enough to avoid interference, then the RFI might be excisable. A number of techniques have been proposed and demonstrated recently [11]-[14]. These fall into three basic categories: temporal, spectral, and statistical some techniques are a combination of two or more. We draw a distinction between operability and excisability when signal analysis beyond conventional radiometric techniques is required. The Aquarius and Hydros approaches to SOE are discussed below.

\section{TERRESTRIAL RADARS}

Long-range radars are required to operate at long wavelengths because of the need to penetrate rain and to use efficient high-power amplifiers [15]. The spectrum from 1215$1400 \mathrm{MHz}(1385 \mathrm{MHz}$ in the U.S. after the year 1999) is allocated for this use. The U.S. operates a number of radars in this band including the FAA Air-Route Surveillance Radars (ARSR-1, -2, -3, and -4) and the Air Force AN/FPS-117 and -24 radars (e.g., [15]-[17]). These radars fall into two general categories: (1) high-power, short pulse, single-frequency and (2) lower-power, longer-pulse, frequency-hopping. The specifications of two representative radars, the ARSR-3 and -4 , are given in Table I. For radiometer measurements with integration times longer than the radar pulse, the amount of interference is affected in part by the energy received. These two radars radiate approximately the same energy as computed by the product of the peak power and the pulse width. Thus, the radar at the higher frequency is more critical. Although the ARSR-4 can operate to $1400 \mathrm{MHz}$, in practice the maximum frequency is less than $1385 \mathrm{MHz}$. Interestingly, the re-allocation of 1385 $1400 \mathrm{MHz}$ from Federal Government use to commercial use was probably indirectly beneficial to using $1400-1427 \mathrm{MHz}$ for passive remote sensing. Using $1385-1400 \mathrm{MHz}$ for receivers requires they be highly-selective (i.e., expensive) to avoid 
TABLE I

PARAMETERS OF TWO FAA AIR-ROUTE SURVEILlaNCE RADARS

\begin{tabular}{|c|c|c|c|c|c|c|c|}
\hline Name & Freq $(\mathrm{MHz})$ & Peak Power (kw) & Ant. Gain (dBi) & Az Beamwidth (deg) & Scan Rate (rpm) & Pulse Width $(\mu \mathrm{s})$ & PRF (Hz) \\
\hline ARSR-3 & $1250-1350$ & 5000 & 34 & 1.25 & 5 & 2 & $310-365$ \\
\hline ARSR-4 & $1215-1400$ & 60 & 35 & 1.4 & 5 & $90+60$ & $216 / 72$ \\
\hline
\end{tabular}

interference from TR's. Thus, the likelihood of widespread use (such as personal communications) is not high, making the spectrum immediately surrounding the EESS passive allocation relatively hospitable.

The amount of interfering power $P_{R F I}$ received by a radiometer is given by the Friis transmission equation:

$$
P_{R F I}=\frac{1}{L_{F D R}} P_{t} G_{t}\left(\theta_{r}, \phi_{r}\right) G_{r}\left(\theta_{t}, \phi_{t}\right)\left(\frac{\lambda}{4 \pi R}\right)^{2}
$$

where $L_{F D R}$ is the frequency-dependent rejection (FDR) factor, $P_{t}$ is the transmit power of the radar, $G_{t}\left(\theta_{r}, \phi_{r}\right)$ is the gain of the radar antenna in the direction of the radiometer, $G_{r}\left(\theta_{t}, \phi_{t}\right)$ is the gain of the radiometer antenna in the direction of the radar, $\lambda$ is the wavelength of the radar frequency, and $R$ is the range between the radar and radiometer. The FDR indicates what fraction of the radar power is available at the radiometer detector. It takes into account both the radiometer passband and the shape of the spectrum transmitted by the radar and is essentially a convolution of the two. The FDR is discussed in detail in ITU-R SM.337-4 Annex 1. The transmit power of the radar can be the radar's peak power, average power, or somewhere in between. We take it to be the peak power and discuss this later in this section. Unless an exact antenna pattern is available, the antenna gain of the radar in the direction of the radiometer can be computed using the ITU recommended pattern (ITU-R 1245.1). This method is both straightforward and conservative from an interference determination perspective. Preliminary antenna patterns for the Hydros and Aquarius radiometers were used for $G_{r}$. Finally, the range $R$ can be calculated knowing the relative positions of the radar and radiometer.

Terrestrial radars transmit pulses with a width $\tau_{p w}$ at a period of $T_{p}$ while the radiometer averages over an integration time $\tau_{\text {int }}$. The differences in these parameters effect the interference-to-noise ratio (INR). For example, consider the case where $\tau_{p w}=2 \mu \mathrm{s}, T_{p}=3 \mathrm{~ms}$, and $\tau_{\text {int }}=1 \mathrm{~ms}$. At most a single radar pulse is received during the radiometer integration period. The time-averaged interference power over an integration period is

$$
\bar{P}_{R F I}=P_{R F I} \cdot \frac{\tau_{p w}}{\tau_{i n t}}
$$

where $P_{R F I}$ is computed using the radar peak power. For short integration times $\left(\tau_{i n t} \leq \tau_{p w}\right)$ the peak power is used. For damage assessment calculation the peak power should also be used. If the radiometer integration time is longer than the radar pulse repetition period, then the average power can be used. The following summarizes these three cases:

$$
\bar{P}_{R F I}=\left\{\begin{array}{cc}
P_{R F I} & \tau_{\text {int }} \leq \tau_{p w} \\
\left(\tau_{p w} / \tau_{\text {int }}\right) P_{R F I} & \text { for } \quad \tau_{p w}<\tau_{\text {int }} \leq T_{p} \\
\left(\tau_{p w} / T_{p}\right) P_{R F I} & \tau_{\text {int }}>T_{p}
\end{array}\right.
$$

The received power can be converted to an equivalent noise temperature using the Nyquist relation for thermal noise power:

$$
\Delta T_{R F I}=\frac{\bar{P}_{R F I}}{k_{B} B}
$$

where $k_{B}$ is Boltzmann's constant and $B$ is the detection bandwidth of the radiometer. We use the $\Delta$-symbol to indicate RFI is an additive effect, always increasing the input-referred power to radiometer. Note, the time-averaged interference power is used so the equivalent temperature is dependent upon the radar and radiometer time characteristics. The INR is defined as the quotient of received interference power and system noise power:

$$
\mathrm{INR}=\frac{\bar{P}_{R F I}}{k T_{s y s} B}
$$

or interference equivalent temperature to system temperature:

$$
\mathrm{INR}=\frac{\Delta T_{R F I}}{T_{\text {sys }}}
$$

where the system temperature

$$
T_{s y s}=T_{a n t}+T_{r x}
$$

is the sum of the interference-free antenna temperature $T_{a n t}$ and the receiver noise temperature $T_{r x}$.

\section{DAmage AND Survivability}

Preventing damage to the radiometer from RFI is the most important mitigation aspect. Because a damaging radar pulse would result in total loss of science, a particularly conservative approach is taken when addressing the issue. The interference power is computed using the following conditions:

1) There is no FDR. The co-channel interference case.

2) The transmit power is the peak power.

3) The transmit antenna gain is its maximum value.

4) The receive antenna gain is its maximum value.

5) The range is the orbital altitude, resulting in the minimum possible path loss.

This set of conditions describes a situation that is essentially a main beam-to-main beam matchup along the nadir-zenith path with the radar transmitting in the radiometer receive band. Although this situation should certainly never occur, it provides built-in margin when determining the maximum 
interference power possible. With the radar allocation extending to $1400 \mathrm{MHz}$ outside the U.S., it is not safe to include any FDR. The main beam-to-main beam matchup can only occur provided the terrestrial radar has a high enough elevation angle and the radiometer has a low enough incidence angle. Otherwise, the link is not quite as intense. One could argue if control of the spacecraft was lost over the right (wrong?) area of the globe the radiometer antenna could point directly down the main beam of a high-power radar. Finally, the range is chosen to be the minimum equal to the orbit altitude just so Friis transmission loss is not responsible for protecting the radiometer from damaging power levels. Given these assumptions, the maximum interfering power for which the radiometer should be protected from damage is:

$$
P_{\text {damage }}=P_{t} G_{t, 0} G_{r, 0}\left(\frac{\lambda}{4 \pi H}\right)^{2}
$$

where $G_{t, 0}$ and $G_{r, 0}$ are the peak antenna gains of the radar and radiometer, respectively; and $H$ is the satellite orbit altitude.

The damage level requirement for the Aquarius and Hydros radiometers [18] is driven by the AN/FPS-108 Cobra Dane radar [16]. The Hydros radiometer design has a $\sim 6-$ meter aperture with $\sim 37 \mathrm{dBi}$ of gain at $1413 \mathrm{MHz}$. The orbit is $\sim 670$ $\mathrm{km}$ above the Earth. The Cobra Dane radar has a peak transmit power of $15.4 \mathrm{MW}$ and an antenna gain of $\sim 47 \mathrm{dBi}$. The damaging threshold for this situation is $2.5 \mathrm{~W}$. The Aquarius radiometer antenna gain is several $\mathrm{dB}$ lower than Hydros and a $2 \mathrm{~W}$ requirement is being levied on the receiver. Both the Aquarius and Hydros designs are such that maximum expected interference will not damage the radiometers.

\section{PRedicted operational LEVEls}

To assess the operability of the radiometer, a Monte-Carlo simulation was run to investigate the interference levels at the radiometer due to a distribution of radars over North America [10]. The simulation included 179 L-band radars distributed as shown in Fig.1. The satellite radiometer, either Hydros or Aquarius, was flown in simulation over the area at the proper altitude and orbit to produce a realistic interference scenario. The simulation was run at 10 millisecond steps with the radar antennas scanning in azimuth, beam-hopping in elevation, frequency-hopping throughout their channel sets, and the Hydros radiometer antenna scanning in azimuth. All three beams of the Aquarius radiometer were modeled. The resulting data were analyzed to determine the interference statistics.

The frequency response of the radiometer and radars was particularly important to this analysis and was captured through the use of the frequency dependent rejection (FDR). FDR's were computed for three different radiometer filters: 7- and 9-pole Chebyshev and a brick-wall (perfect) with 25$\mathrm{MHz}$ bandwidth. The Radar Spectrum Engineering Criteria spectrum (RSEC) mask shape [1, Ch.5] was used for the radars. The FDR for the three radiometer filters against the emission envelope of the AN/FPS-117 radar are shown in

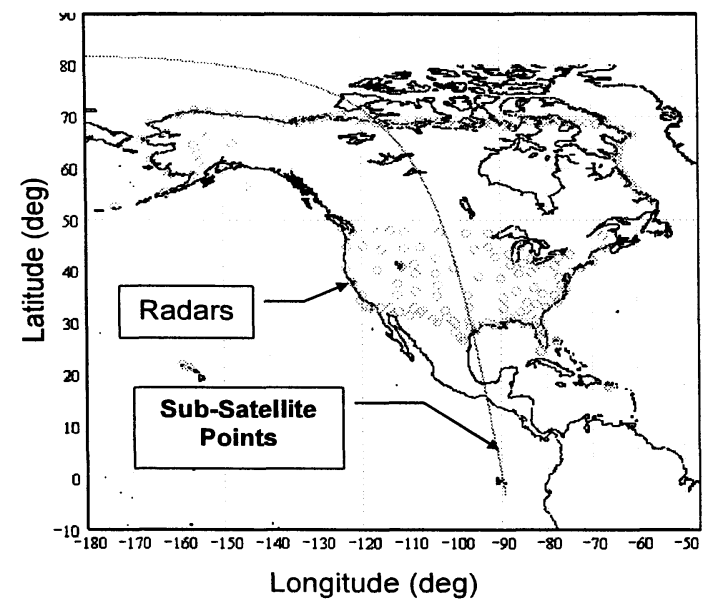

Fig. 1. Map of radar locations and sub-satellite track for Hydros interference analysis.

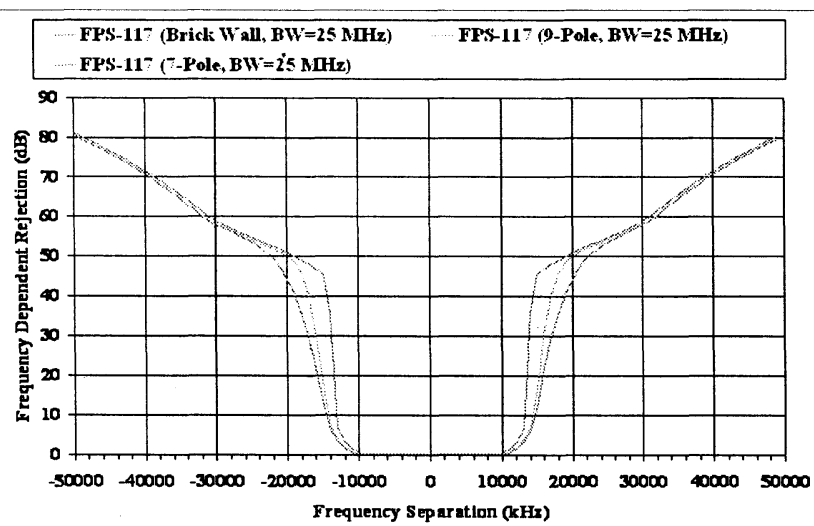

Fig. 2. Frequency depedence rejection (FDR) for three different radiometer filters and AN/FPS-117 emission spectrum envelope.

Fig.2. The minimum frequency separation is $28 \mathrm{MHz}$ under the reallocated spectrum rules (maximum radar frequency of $1385 \mathrm{MHz}$ ). Note, there is no difference in rejection at $28 \mathrm{MHz}$ separation for the different radiometer filters. This is because the floor of the RSEC mask shape dominates the rejection for frequency separations away from the filter skirts. While this approach is conservative, there is evidence that in practice radar pulses occupy a large portion of spectrum.

Once an over-pass is simulated, the results are compiled into a histogram and resulting cumulative distribution function (CDF). These can be used to determine data availability given a threshold interference level. According to Recommendation ITU-R SA.1028-2 "Performance Criteria for Satellite Passive Remote Sensing" the recommended data availability for L- 


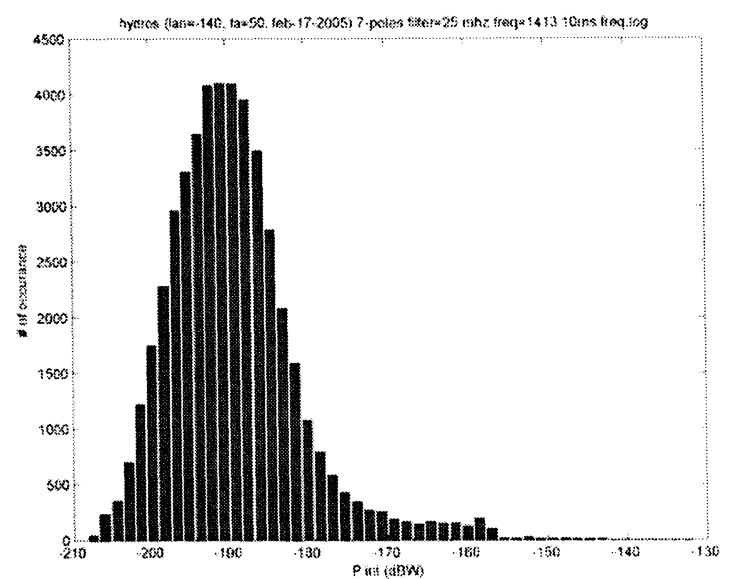

(a)

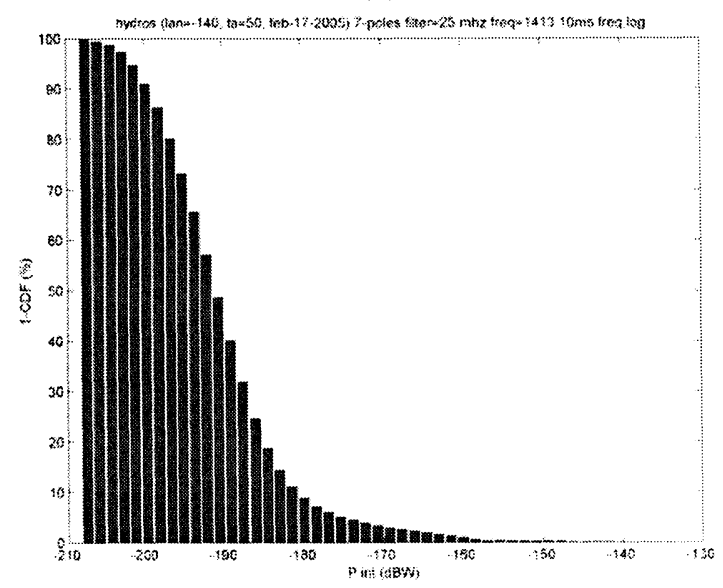

(b)

Fig. 3. Histogram and $\mathrm{CDF}$ of simulated interference power received by Hydros over Alaska.

band observations is $99.9 \%$ and according to Recommendation ITU-R SA.1029-2 "Interference Criteria for Satellite Passive Remote Sensing" the recommended maximum interference level is $-174 \mathrm{dBW}$. This power level is equal to $0.01-\mathrm{K}$ equivalent noise temperature increase over a $27-\mathrm{MHz}$ bandwidth. The CDF (actually 1-CDF) for a Hydros pass over Alaska using a 7-pole radiometer filter is shown in Fig.3. The interference over Alaska is due to a group of several AN/FPS-117 radars and the $-174 \mathrm{dBW}$ interference threshold is exceeded $1 \%$ of the time. The $0.1 \%$ permissible interference fraction occurs at about $-164 \mathrm{dBW}$ (or $0.1 \mathrm{~K}$ ). Simulations for Aquarius were also run over the Pacific and Atlantic Oceans and indicate the interference threshold is only exceeded $0.05 \%$ of the time, within the recommended data availability.

\section{EXCISABILITY}

For the $1 \%$ of cases in which the radar interference is not controllable with filtering, excision is desirable. If the radar pulses are detectable in the radiometer data, the offending samples can be dropped from processing to effectively remove the RFI. Pulse detection and time-blanking has been shown to be an effective mitigation approach [12] in radio astronomy. The kurtosis method [14] is another possibility for detection, however it was at too low of a technology readiness level (TRL) for Aquarius (and was for Hydros at the time of formulation). To detect radar pulses the radiometer integration time must be short enough to capture a pulse and long enough to minimize random noise. An integration time equal to the pulse width, approximating a matched filter, would be ideal. For data volume reasons this, however, might not be desirable. The trade-off of integration time versus detectability can be illustrated by a detection plot - one for the ARSR-4 is shown in Fig.4. The plot shows interference contours for different values of peak interference $P_{R F I}$. The contours are generated using (3) plus an additional accounting for the sweep of the TR main beam at radiometer integration times greater than the dwell time of the radar beam:

$$
\bar{P}_{R F I}\left(\tau_{i n t}>\theta_{a z} / \omega\right)=\frac{\theta_{a z} / \omega}{\tau_{i n t}} \frac{\tau_{p w}}{T_{p}} P_{R F I}
$$

where $\theta_{a z}$ is the TR azimuth beamwidth and $\omega$ is the rotation rate. The four dashed, diagonal lines are the $0.1,1$, 3 , and 5 times contours of $\left(B \tau_{\text {int }}\right)^{-1 / 2}$ for $B=25 \mathrm{MHz}$. Radar interference located above the highest dashed line are detectable with a $0.00003 \%$ false-alarm rate and the second highest with a $0.135 \%$ false-alarm rate. There are two knees that are local maxima of detectability: one at $\tau_{i n t}=\tau_{p w}$ and the other at $\tau_{\text {int }}=\theta_{a z} / \omega$. Integrating longer causes the INR to drop as $\tau_{\text {int }}^{-1}$ while the noise only drops as $\tau_{\text {int }}^{-1 / 2}$. Conversely, integrating less does not increase the INR but only increases the noise. These results should not be surprising as integrating over the radar pulse (or main beam sweep) approximates a matched-filter receiver for maximum detectability. With these results in mind, the Hydros radiometer is designed to integrate between its on-board radar pulses with no additional averaging resulting in $\tau_{\text {int }} \approx 250 \mu \mathrm{s}$. This is possible because of the large amount of on-board storage available on Hydros. The Aquarius radiometer, however, is memory limited so it's maximum integration time is required to be less than $30 \mathrm{~ms}$.

\section{CONCLUSION}

In this paper, three aspects of RFI mitigation in microwave radiometers were discussed: survivability, operability, and excisability (SOE). Application was made to NASA's Hydros and Aquarius radiometers. For survivability, it was found the Hydros radiometer must be protected against $2.5 \mathrm{~W}$ of interference power in a worst case scenario. Because the antenna is slightly smaller, a $2 \mathrm{~W}$ requirement is being used for Aquarius. Different filter responses were used to study the frequency dependent characteristics of interference. It was shown a 7pole Chebyshev response performs no worse than a perfect brick-wall filter at rejecting radar interference. Simulations of satellite over-passes indicate the Aquarius radiometer using a 7-pole equivalent filter will experience interference exceeding 


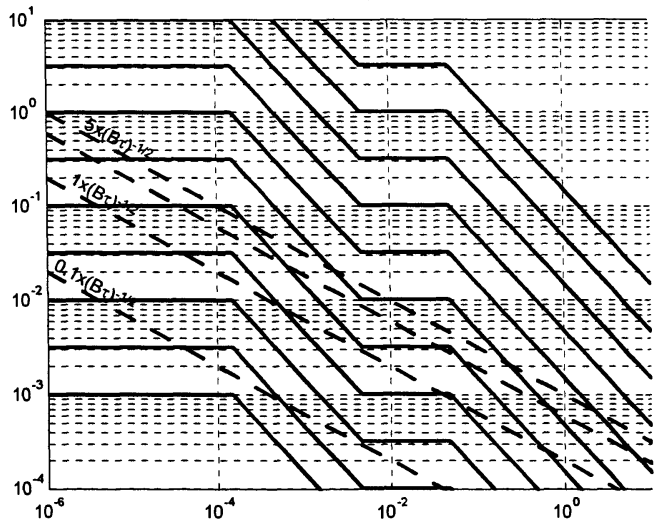

Fig. 4. Plot showing INR contours for constant peak interference (solid lines) and radiometer noise contours $\alpha\left(B \tau_{\text {int }}\right)^{-1 / 2}$ for $B=25 \mathrm{MHz}$ and $\alpha=0.1,1,3$, and 5 (dashed lines). Radar interference is detectable when a solid line is above a dashed line for desired criteria.

the recommended $-174 \mathrm{dBW}(0.01 \mathrm{~K})$ no more than $0.05 \%$ of the time over ocean. The Hydros radiometer simulations resulted in a $1 \%$ exceed rate over Alaska. The $0.01 \%$ rate occurs at $-164 \mathrm{dBW}(0.1 \mathrm{~K})$. Finally, the potential to detect and blank radar pulses or main beam sweeps was investigated. As a result, requirements were imposed on the Aquarius radiometer integration time to maximize the probability of detection. In conclusion the use of the SOE criteria has resulted in concrete requirements and design measures being taken in the Aquarius and Hydros radiometer developments.

\section{ACKNOWLEDGMENTS}

The authors thank John Zuzek of NASA's Spectrum Management Office for taking an interest in our work and providing for the RFI simulations to be performed. Special thanks go to Rafat Nasser, Mark Burns, and Ron Lepowski of ITT Industries for performing the simulations and providing in depth knowledge of terrestrial radars.

\section{REFERENCES}

[1] NTIA Manual of Regulations and Procedures for Federal Radio Fre quency Management. U.S. Department of Commerce: U.S. Government Printing Office S/N 903-008-00000-8, 2000, available on the World Wide Web at http://www.ntia.doc.gov/osmhome/redbook/redbook.html.
[2] D. Entekhabi, E. G. Enjoku, P. Houser, M. Spencer, T. Doiron, Y. Jim, J. Smith, R. Girard, S. Belair, W. Crow, T. J. Jackson, Y. H. Kerr, J. S. Kimball, R. Koster, K. C. McDonald, P. E. OŠNeill, T. Pultz, S. W. Running, J. Shi, E. Wood, , and J. van Zyl, "The hydrosphere state (Hydros) satellite mission: An earth system pathfinder for global mapping of soil moisture and land freeze/thaw," IEEE Trans. Geosci. Remote Sensing, vol. 42, no. 10, pp. 2184-2195, October 2004

[3] D. L. Vine, G. Lagerloef, F. Colomb, and Y. Chao, "Aquarius : A Passive/Active microwave sensor to monitor sea surface salinity globally from space," Microwave Remote Sensing of the Environment IV, Proc. SPIE, vol. 5654, pp. 122-128, 2004.

[4] Y. Kerr, P. Waldteufel, J. Wigneron, J. M. A. J. Font, and M. Berger "Soil moisture retrieval from space: The Soil Moisture and Ocean Salinity (SMOS) mission," IEEE Trans. Geosci. Remote Sensing, vol. 39, no. 8, pp. 1729-1735, August 2001.

[5] D. LeVine, "ESTAR experience with RFI at L-band and implications for future passive microwave remote sensing from space," in Proc. Int. Geosci. and Remote Sens. Symp. (IGARSS), Toronto, ON, Canada, 2002, pp. 847-849.

[6] D. LeVine and M. Haken, "RFI at L-band in synthetic aperture radiometers," in Proc. Int. Geosci. and Remote Sens. Symp. (IGARSS), Toulouse, France, 2003, pp. 1742-1744.

[7] S. Ellingson, G. Hampson, and J. Johnson, "Characterization of L-band RFI and implications for mitigation techniques," in Proc. Int. Geosci. and Remote Sens. Symp. (IGARSS), Toulouse, France, 2003.

[8] J. Piepmeier, O. Ugweje, M. Midon, and A. Caroglanian, "Radio frequency survey of the $21-\mathrm{cm}$ wavelength $(1.4 \mathrm{GHz})$ allocation for passive microwave observing," in Proc. Int. Geosci. and Remote Sens. Symp. (IGARSS), Toulouse, France, 2003, pp. 1739-1741.

[9] A. Camps, I. Corbella, F. Torres, J. Bara and J Capdevila "RF interference analysis in aperture synthesis interferometric radiometers: Application to L-band MIRAS instrument," IEEE Trans. Geosci. Remote Sensing, vol. 38, no. 2, pp. 942-950, 2000.

[10] R. Nasser, "Analysis of ground-based radar power levels at EESS sensors in the 1400-1427 MHz band," NASA's Goddard Space Flight Center Tech. Rep., 2004, government point-of-contact is Jeffrey Piepmeier at NASA's Goddard Space Flight Center.

[11] A. Gasiewski, M. Klein, A. Yevgrafov, and V. Leuskiy, "Interference mitigation in passive microwave radiometry," in Proc. Int. Geosci. and Remote Sens. Symp. (IGARSS), Toronto, Canada, 2002, pp. 1682-1684.

[12] S. Ellingson and G. Hampson, "Mitigation of radar interference in 1-band radio astronomy," Astrophysical J. Supp., vol. 147, no. 1, pp. 167-176, 2003.

[13] J. T. Johnson, G. A. Hampson, and S. Ellingson, "Design and demonstration of an interference suppressing microwave radiometer," in Proc. Int. Geosci. and Remote Sens. Symp. (IGARSS), Anchorage, Alaska, 2004, pp. 1683-1686.

[14] C. Ruf, S. Gross, and S. Misra "RFI detection and mitigation for microwave radiometry with an agile digital detector," IEEE Trans. Geosci. Remote Sensing, vol. 44, no. 3, pp. 694-706, 2006.

[15] J. E.F. Drocella, S. Jones, and J. W.T. Druhan, "Spectrum reallocation report: Response to title III of the balanced budget act of 1997," National Telecommunications and Information Administration, Tech. Rep. NTIA Special Publication 98-36, 1998

[16] M. Skolnik, Radar Handbook, 2nd ed. New York: McGraw-Hill, 1990.

[17] R. Lay, J. Taylor, and G. Brunins, "ARSR-4: Unique solution to long recognized radar problems," in Proc. IEEE Int. Radar Conf., Arlington, Virginia, 1990, pp. 6-11.

[18] M. Spencer, E. Njoku, D. Entekhabi, T. Doiron, J. Piepmeier, and R. Girard, "The Hydros radiometer/radar instrument" in Proc. Int. Geosci. and Remote Sens. Symp. (IGARSS), Anchorage, Alaska, 2004. 